\title{
BMJ Open Is care based on comprehensive geriatric assessment with mobile teams better than usual care? A study protocol of a randomised controlled trial (The GerMoT study)
}

\author{
Anne Wissendorff Ekdahl, ${ }^{1,2}$ Anna Axmon, ${ }^{3}$ Magnus Sandberg, ${ }^{4}$ \\ Katarina Steen Carlsson ${ }^{5}$
}

To cite: Ekdahl AW, Axmon A, Sandberg M, et al. Is care based on comprehensive geriatric assessment with mobile teams better than usual care? A study protocol of a randomised controlled trial (The GerMoT study). BMJ Open 2018;8:e023969. doi:10.1136/ bmjopen-2018-023969

- Prepublication history for this paper is available online. To view these files please visit the journal online (http://dx.doi org/10.1136/bmjopen-2018023969).

Received 6 May 2018

Revised 27 July 2018

Accepted 13 September 2018

Check for updates

(c) Author(s) (or their employer(s)) 2018. Re-use permitted under CC BY-NC. No commercial re-use. See rights and permissions. Published by BMJ.

For numbered affiliations see end of article.

Correspondence to Dr Anne Wissendorff Ekdahl; anneekdahl@gmail.com

\section{ABSTRACT}

Introduction Comprehensive geriatric assessment (CGA) is a multidimensional, interdisciplinary diagnostic process used to determine the medical, psychological and functional capabilities of frail older people. The primary aim of our current study is to confirm whether CGA-based outpatient care is superior than usual care in terms of health-related outcomes, resource use and costs.

Methods and analysis The Geriatric Mobile Team trial is designed as a single-centre randomised, controlled, assessor-blinded (at baseline) trial. All participants will be identified via local healthcare registries with the following inclusion criteria: age $\geq 75$ years, $\geq 3$ different diagnoses and $\geq 3$ visits to the emergency care unit (with or without admittance to hospital) during the past 18 months. Nursing home residency will be an exclusion criterion. Baseline assessments will be done before the 1:1 randomisation. Participants in the intervention group will, after an initial CGA, have access to care given by a geriatric team in addition to usual care. The control group receives usual care only. The primary outcome is the total number of inpatient days during the follow-up period. Assessments of the outcomes: mortality, quality of life, health care use, physical functional level, frailty, dependence and cognition will be performed 12 and 24 months after inclusion. Both descriptive and analytical statistics will be used, in order to compare groups and for analyses of outcomes over time including changes therein. The primary outcome will be analysed using analysis of variance, including in-transformed values if needed to achieve normal distribution of the residuals.

Ethics and dissemination Ethical approval has been obtained and the results will be disseminated in national and international journals and to health care leaders and stakeholders. Protocol amendments will be published in ClinicalTrials.gov as amendments to the initial registration NCT02923843. In case of success, the study will promote the implementation of CGA in outpatient care settings and thereby contribute to an improved care of older people with multimorbidity through dissemination of the results through scientific articles, information to politicians and to the public.

Trial registration number NCT02923843; Pre-results.
Strengths and limitations of this study

- Uses randomised design in a well-defined population.

- Addresses the urgent need of better care models for older people with high healthcare utilisation.

- Uses highly personalised care (the intervention) which cannot be fully standardised.

The single-centre design limits generalisation.

\section{INTRODUCTION}

\section{Background}

With the ageing of populations worldwide, increasing numbers of people are living with multiple chronic conditions and frailty. ${ }^{1}$ However, our healthcare system is not optimally designed to meet the complex needs of older people with multimorbidity. Instead, it has been subdivided into an ever-increasing number of entities and specialities over the last decades. Thus, the development of healthcare has led care providers to focus on the treatment of single diseases instead of addressing multimorbidity. As a consequence, the care of old people has become more fragmented leading to increased risks, such as medication errors. ${ }^{1}$ In addition, the current health system is associated with high costs because of repeated visits to emergency care units and hospitalisation of older people. ${ }^{2}$

Comprehensive geriatric assessment (CGA) is a multidimensional, interdisciplinary diagnostic process used to determine the medical, psychological and functional capabilities of frail older people ${ }^{3}$ and includes further planning and follow-up of the patients. ${ }^{3}$ Evidence suggests that CGA-based care is superior to usual care in terms of improving functional capacity and reducing the risk of institutionalisation. ${ }^{34}$ 
Older people with multimorbidity often require hospital care to optimise the treatment of chronic diseases or to diagnose and treat newly diagnosed conditions. ${ }^{2}$ At the same time, hospitalisation may serve as a marker of unmet healthcare needs and should, if possible, be avoided due to the associated risks of fractures, falls, medication errors, delirium, iatrogenic infections and further disabilities. ${ }^{5}$

There have been several studies and meta-analyses on CGA-based care compared with usual care in the acute inpatient care setting, ${ }^{346-8}$ but only a few randomised controlled trials on the effect of CGA-based outpatient care. ${ }^{9}{ }^{10}$ Boult $e t$ a ${ }^{9}$ showed better functionality but no difference in mortality with CGA-based care. Our own previous study, the Ambulatory Geriatric- Frailty Intervention Trial (Age-FIT) trial, showed superior results of CGA-based care compared with usual care with respect to days in hospital, feeling of security and mortality. ${ }^{11}$ In addition, results from the same study indicated a reduction of progression in frailty. ${ }^{12}$

Despite the evidence behind CGA-based care, healthcare providers have been reluctant to adopt this method, probably due to the anticipation of increased costs and the need for substantial shifts in practice towards interprofessional teamwork-including gerontological and geriatric competences. The study will therefore include health economic data from both inpatient and outpatient's healthcare and care given by the municipality, which makes it possible to evaluate the cost-effectiveness of the study.

By giving patients in the intervention group (IG) easy access to care given by a team who knows them well, we hope, as in our former study, we will be able to diminish the need of inpatient days in hospital which is an important outcome because of the risk for delirium, falls, infections and other iatrogenic complications associated with hospitalisation of older people ${ }^{13}$ beside high healthcare costs.

We used the Standard Protocol Items: Recommendations for Interventional Trials checklist when writing our report. $^{14}$

\section{METHODS AND ANALYSES \\ Trial design}

The study is designed as a randomised, controlled, assessor-blinded, single-centre trial. Participants are randomised to one of two groups; an IG receiving care of the CGA-based team in addition to usual healthcare and a control group (CG), with access to usual healthcare only. The study will be conducted at a medium-sized non-academic hospital in the south of Sweden. The hospital has approximately 140000 inhabitants to serve. The care will be described through reviews of registries as well as the case report form (CRF). The care registries are: 'The Patient Administrative System in Skane' (PASIS),${ }^{15}$ which is a population-based, administrative database run by the County Council of Skane together with the database of decisions according to the Social Care Act in Sweden run by the Swedish National Board of Health and Welfare. ${ }^{16}$
PASIS gives the base for all funding and follow-up of care in Skane. Both registries use the specific person-identification number which all inhabitants in Sweden have.

\section{Patient and public involvement}

The base for the interventions was founded through several meetings with the Swedish retirements organisations and three medical societies (general practitioners, specialists in internal medicine and geriatricians). The meetings resulted in a statement outlining best way to take care of older people with multimorbidity: 'Around every frail older person there should be a multiprofessional team adapted to the specific needs of that person. The team should coordinate the care around the frail older person'.

This project aims to fulfil that goal-to provide with a multiprofessional team adapted to each patients' needs. The patients were, however, not involved in the study design, the recruitment or the choice of outcomes. The burden of the intervention was not assessed by the patients before the start of the study, but it was explained in detail in the study information before any agreement of participation.

The results of the study will be expressed in a reader-friendly version and mailed out to all study participants.

\section{Setting}

The hospital admits surgical and medical emergencies 24 hours a day. The Geriatric Mobile Team (GerMoT) is located in a geriatric department and several team members work both in the mobile team and in other parts of the department. Apart from the GerMoT, there is no general geriatric outpatient clinic and no private geriatric practitioners in the municipality.

\section{Eligibility}

Eligible participants will

1. have had $\geq 3$ visits to the emergency care unit within the past 18 months.

2. have $\geq 3$ different diagnoses according to the International Statistical Classification of Diseases and Related Health Problems, 10th revision.

3. be living in, or close to, the municipality in which the hospitals are situated.

4 . be $\geq 75$ years old.

5. not be living in a nursing home.

In this study, we have chosen to exclude persons living in nursing homes as they receive healthcare by designated primary care physicians who make weekly rounds at the respective accommodations. Moreover, we decided to only include subjects residing in municipalities located in the vicinity of the hospital (as one of our inclusion criteria is based on the frequency of the subjects having attended the emergency care unit).

Importantly, we did not exclude participants with cognitive decline as we know that these individuals often seek care and that their cognitive decline is frequently not addressed properly. ${ }^{17}$ 
In the present study, we seek to confirm our earlier results with a slightly modified recruitment scheme $(\geq 3$ visits to the emergency care unit, with or without subsequent admittance).

\section{Outcomes}

Primary outcome

- Total number of days of inpatient care days during 24 months obtained from the registers of care in Region Skane, PASIS. This registry will capture all kinds of care in the Region of Skane given by both private and public care in the Region-not only care given in the hospital in which the intervention is placed. The number of inpatient days are strictly defined as more than 24 hours of inpatient care.

\section{Secondary outcomes}

- Mortality after 12 months (security data) and after 24 months (obtained from the Swedish National Population Register).

- Quality of life, as measured by the EQ-5D-5L ${ }^{18}$ at baseline, after 12 and 24 months

- Healthcare use (obtained from the local care register, PASIS, where contacts with primary and secondary care-including visits to nurses, paramedics, physicians etc-are registered, together with data from the registry of social care from the National Board of Health and Welfare, which describes the social service interventions during the study period).

- Physical function level (obtained by SPPB) ${ }^{19}$ at baseline, after 12 and 24 months.

- Frailty, as measured according to the Fried (phenotype) and Rockwood's deficit index of frailty at baseline, after 12 and after 24 months ${ }^{20} 21$

- Dependence measured with Katz' Activities of Daily Living at baseline, after 12 and 24 months ${ }^{22}$

- Cognition, as measured with Montreal Cognitive Assessment at baseline and after 24 months ${ }^{23}$

Background variables will be the following sociodemographic factors: age, sex, marital status, living alone/in a relationship and education - as they are all known to be associated with health status.

Data will be collected by means of structured interviews at baseline, after 12 and 24 months as well as by collection of registry data. Registry data will be collected for the period 4 years before inclusion in the study to 24 months after inclusion. Mortality data will be analysed for the first 12 months after inclusion for security reasons.

\section{Participant timeline}

For the timeline, please see research checklist.

\section{Sample size}

A power calculation was made based on the primary outcome variable, that is, mean number of days in hospital. Based on a former similar study, there will be an assumed difference between the IG and the CG of 4.1 days in hospital during the 24-month study period (11.1 days in the IG and 15.2 in the CG) with an SD of 15 days in both groups. To be able to detect a difference between the IG and the CG with a two-sided test and with a significance level of $\alpha=0.05 \%$ and $80 \%$ power, at least 211 participants in each group will be needed. There will be almost no loss to follow-up of the primary outcome based on previous research of this patient group and intervention. ${ }^{10}$ Thus, a total of 450 persons will be included.

\section{Recruitment}

Lists of eligible participants will be obtained from the Healthcare Administrative System (PASIS) in Region Skane. To ensure as many participants as possible, all eligible individuals will receive a letter with an explanation of the aim and procedure of the study about a week before they are contacted via phone by an experienced study nurse (the head researcher nurse). If requested, further information can be given at this point. In case of a preliminary consent, the participants' address and phone numbers are given to the data-collecting nurses who will make an appointment for a home visit, during which the written consent will be obtained before the baseline data are collected. If a person due to cognitive decline is unable to answer the study questions or give informed consent, a proxy will be contacted.

Unreachable eligible participants will be contacted at least three times before we give up contacting them.

\section{Allocation}

Prior to inclusion of the first patient, a randomisation master list with 450 numbers (the number needed according to the power calculation) was created by the project coordinator via a computer package (IBM SPSS Statistics V.23.0). The participants were randomised 1:1 to either the IG or the CG.

The list is kept by a study administrator who is not involved in the recruitment of participants.

When informed consent and baseline measurements have been collected, the protocol will be delivered to the study administrator who randomises the participants consecutively as the protocols become available to her. After randomisation, the participants will receive an information letter describing the study conditions for that group. In addition, a nurse from GerMoT will get in contact with each participant allocated to the IG to arrange for further procedures as described in the intervention. Similarly, the participants in the $\mathrm{CG}$ will receive a letter explaining the study conditions and that they will be contacted again after 12 and 24 months, respectively.

Only the project coordinator and the study administrator have access to the randomisation list during the study.

\section{Blinding}

All prerandomisation baseline data are collected by blinded assessors/interviewers, who will not take part in the patient's care before or after randomisation. The participants in this study cannot be blinded to their assigned groups at 12 and 24 months, as only participants 
in the IG are assessed and cared for by the GerMoT. All register data will be extracted by blinded administrative personnel not involved in the study.

\section{Data collection}

All instruments in the questionnaires are validated (see references in the Outcomes section. ${ }^{18} 19$ 21-23 All data collectors are registered nurses who have been trained in good clinical practice (GCP). There will be regular meetings between the data collectors, the head researcher nurse and the project leader. Such meetings will be held primarily to provide training for all instruments and later also to address all upcoming questions. All paper protocols will be kept safe and when data are transferred to a computerised database, the questionnaires will be checked for errors and missing data by research staff. Data entry are double-checked against the paper questionnaires, and in case of inconsistencies in data entries, corrections will be made by referring back to the paper questionnaires.

Through the 10-digit unique personal identification number provided to all people in Sweden, it will be possible to extract register data on all participants who have provided informed consent, even in case they have died.

\section{The intervention}

The key component of the intervention is a CGA, including a plan for future care and follow-up contacts. All care is personalised and adapted to each individual's needs. The following scheme will be used for most of the participants (exceptions can be made, eg, if it is difficult for the participant to visit the hospital):

1. Home visit by a nurse: An interview regarding the participant's own view of his or her health and health problems. The interview is conducted by a nurse in the participant's home environment as it gives us a better opportunity to further deepen our understanding of the participant's living conditions. The interview has a holistic character and follows a template that covers areas closely related to the patient's quality of life. In addition, a venous blood sample is drawn and the following parameters will be analysed: haemoglobin, white cell count and platelet count, proB-type natriuretic protein, blood glucose, creatinine, sodium, potassium and C-reactive protein.

2. Drug review by a clinical pharmacist: A thorough drug review is performed together with the participants and their relatives as well as via the pharmacies' national registries and the participants' medical records. Potentially harmful drugs or doses are being revised. Finally, this information is assembled into the medical record.

3. Visiting a physician in hospital: Prior to the visit, the physician performs a thorough review of the medical records. This information is assembled into one comprehensive medical history entry. When visiting the physician, the participant is asked to bring a relative or a close friend. At this time, the medical history is con- firmed, current health symptoms are listed and a physical examination, including neurological assessment and an ECG, is performed. The participant receives information about the outcome of the blood analyses. The visit ends with a summary of all assessments, adjustment of the current medication list and a plan for the next contact, which will usually take place via telephone by a nurse or another team member some weeks after the interdisciplinary meeting conference. The patient is handed a printout of his or her current medication list (or one will be sent by mail within the following days).

4. Interdisciplinary meeting conference: Such conferences are held twice a week by the CGA team. The team includes nurses, physicians, a physiotherapist, an occupational therapist and a pharmacist. In addition, social workers from the municipality will attend. The team summarises the participants' physical, psychological, social and functional situation, with a focus on actions to improve quality of life. A plan for further actions is taken if possible and necessary. Examples of such actions include home visits by occupational therapists or physiotherapists as well as further pharmaceutical adjustments or new contacts with the municipal liaison for reassessment of service needs. Decisions are also made on the type and frequency of the follow-up contacts. Importantly, the plan can vary widely between participants and range from a short period of daily contacts with the nurse and extra doctor's visits, to scheduled 6-monthly phone calls or visits after 1 year by a nurse (in cases where no earlier contact is deemed necessary).

\section{Accessibility}

The GerMoT office is open for telephone calls during office hours (weekdays 08:00-16:30) and typically one of the nurses will answer the participants' calls. If the nurses are unable to answer, the participant has the opportunity to leave a recorded message, on which the nurse will return the call on the same day. The GerMoT instructs the participants to contact the regular emergency healthcare services whenever there is a need outside office hours. In cases of uncertainty whether to contact emergency services during office hours, patients are encouraged to call the GerMoT first. In case of an urgent health-related problem, the GerMoT can guide the participant to the appropriate caregiver. The GerMoT will have no formal right to admit a patient directly to a particular department of the hospital but will, whenever needed, consult with appropriate specialist colleagues to make the best possible arrangements.

\section{Collaboration and coordination}

The GerMoT must cooperate extensively with other healthcare providers, as it is regarded as an adjunct to the existing healthcare system. If patients have contacts with several physicians in parallel, especially in primary care, these are contacted in order to minimise the risk 
of uncoordinated care. The primary care-based management of certain diseases will also be available to the participants in the IG. For example, health services for diabetes, chronic obstructive pulmonary disease and skin ulcers will be continually provided by specialised nurses at the primary care centres. The physicians and coordinator nurses at the GerMoT have regular contact with these nurses as deemed appropriate. For organ-specific consultations, the physicians and nurses of the GerMoT contact consultant physicians at relevant hospital departments.

\section{Usual care}

Participants allocated to the CG receive healthcare either from their primary care physician, the community services or the inpatient and outpatient hospital care. Normally, most primary care is provided at the request of the patients-and only more seldom in the form of prescheduled proactive health visits. Both the IG and the CG have access to the primary care centres, the hospital and various ambulatory units on equal conditions. The GerMoT concept is not part of the regular healthcare system, where the patients instead are seeking one professional at a time-for example, either a physician, a nurse or a physiotherapist.

\section{Criteria for discontinuing or modifying the allocated interventions}

A participant may at any time leave the trial, without having to explain their decision. If a patient moves to a nursing home, the intervention given by the GerMoT will end at the same time as the physician in the nursing home takes over the responsibility for coordination of the medical care. The intervention will also be stopped if a patient moves out of the hospital's catchment area.

\section{Strategies to improve adherence to the intervention}

Adherence to the intervention is promoted by proactive calls by the GerMoT nurse, especially if there are reasons to believe that the participant does not seek appropriate care on his or her own initiative.

In addition to the service provided by the GerMoT, care is permitted without restrictions.

\section{Statistical methods}

Both descriptive and analytical statistics will be used in order to compare groups and for analyses of outcomes over time including changes therein.

Continuous outcomes, for example, days in hospital, will be analysed using analysis of variance, including in-transformed values if needed to achieve normal distribution of the residuals. Dichotomous outcomes will be evaluated using relative risks estimated by generalised linear models with a Poisson distribution, log link function and robust covariance matrix estimator. Outcomes described by ordinal data will be investigated using non-parametric statistical methods, such as Pearson's $\mathrm{X}^{2}$ test.

A two-sided $p$ value $<0.05$ will be considered statistically significant. Analyses will be made on the basis of the intention-to-treat principle. Given the old age of the participants, a relatively high dropout rate is expected, and missing data will not be at random. Simply analysing complete cases is not relevant and might lead to bias. Therefore, the approach to data imputation will be the replacement of missing values with a value based on the median change of deterioration. A worst case change will be applied for those who have died before follow-up.

The first protocol V.1.6 was launched on the 14 October 2016. This article is based on protocol V.1.7. launched on the 24 April 2017. Changes can be followed in ClinicalTrials.Gov and the main change between V.1.6 and V.1.7 was that we lowered the recruitment age to $\geq 75$ years as in our previous study instead of $\geq 78$ years. This was done to allow recruitment of a sufficient number of eligible participants.

The funder of the clinical part of this trial is Region Skane, Sweden. For the scientific part, the primary sponsor is the first author and principal investigator (PI) Anne W Ekdahl, anneekdahl@gmail.com, cell 04670787 4250.

Participants are still being recruited to the trial. The first patient was enrolled on the 26 October 2016 and the last is expected to be enrolled in June 2018. After the recruitment of the last patient, the trial will go on for a further 24 months and is therefore expected to end in the summer of 2020.

All items asked for in the WHO Organisation Trial Registration Data Set can be found in the ClinicalTrials. Gov: NCT02923843 together with the information on this page.

\section{ETHICS AND DISSEMINATION}

The study results will be disseminated in national and international scientific peer-reviewed journals and on appropriate congresses. The results will also be disseminated to healthcare leaders and stakeholders.

Ethical approval has already been obtained.

Protocol amendments will be published in ClinicalTrials.gov as amendments to the initial registration NCT02923843.

An interim analysis will be made on mortality after 1 year. This will be done by an independent researcher and the study will be stopped in case of a statistically significant increase or decrease in mortality. The independent researcher must in that case inform the project leader who will then terminate the trial.

There is no specific data monitoring committee besides the above-mentioned mortality analysis as we are using no new methods in the CG or IG which could be considered harmful to the patient.

\section{Data management and monitoring}

All participants are given a code number between 1 and 450. The master randomisation list is safely stored by the study administrator. All case report forms (CRFs) with code numbers are safely locked in and stored in a locked cabinet in the head research nurse's office. The 
initial entry of data will be made in a computerised database constructed by an experienced statistician able to promote data quality by allowing data entry within certain ranges. The study conducted at the centre in Region Skane will be monitored by the Clinical Studies Sweden-Forum South after baseline, 12 and 24 months. This organisation has no connection to the study besides quality assurance of clinical trials conducted by Lund University and in Region Skane.

The GCP-trained head researcher nurse will first obtain a verbal consent and later the data-collecting nurses will obtain written consent during the home visits to each participant. In case of language problems or cognitive decline, a next of kin can sign the written informed consent-preferably together with the participant.

All CRFs will be kept in a locked cabinet at the head research nurse's office during the trial. However, at the 12 months and the 24 months monitoring, independent staff will be allowed to access all the CRF, randomisation list and medical records.

The PI and the authors of relevant articles will have access to the data set. Further dissemination of the data set can be decided by the PI.

\section{Author affiliations}

${ }^{1}$ Division of Clinical Geriatrics, Department of Neurobiology, Care Sciences and Society (NVS), Karolinska Institute (KI), Stockholm, Sweden

${ }^{2}$ Department of Clinical Sciences Helsingborg, Geriatric Medicine, Lund University, Helsingborg Hospital, Stockholm, Sweden

${ }^{3}$ Division of Occupational and Environmental Medicine, Department of Laboratory Medicine, Lunds University, Lund, Sweden

${ }^{4}$ Department of Health Sciences, Lunds Universitet, Lund, Sweden

${ }^{5}$ The Swedish Institute for Health Economics, Lund, Sweden

Acknowledgements A special thanks to Einar Rystedt, MD, and professor Martin Ingelson, $\mathrm{MD}, \mathrm{PhD}$, at Uppsala University for helpful comments on the manuscript.

Contributors AWE designed the study and wrote this manuscript. AA and KSC participated in designing the study, with a focus on statistics and health economy, respectively. All authors, AWE, AA, KSC and MS, have scrutinised, improved and approved the final manuscript.

Funding The writing of this study protocol article received grants from the Zoega Foundation (grant number TZ2016-0012) and the Gorthon Foundation (grant number 2016-1289). The full RCT will be funded by Region Skane and the Kamprad Foundation (grant number 20170077). Additional grants will be applied for in the future.

Competing interests None declared.

Patient consent Obtained.

Ethics approval Regional Ethical Committee in Lund Dnr: 2016/630.

Provenance and peer review Not commissioned; externally peer reviewed.

Open access This is an open access article distributed in accordance with the Creative Commons Attribution Non Commercial (CC BY-NC 4.0) license, which permits others to distribute, remix, adapt, build upon this work non-commercially, and license their derivative works on different terms, provided the original work is properly cited, appropriate credit is given, any changes made indicated, and the use is non-commercial. See: http://creativecommons.org/licenses/by-nc/4.0/.

\section{REFERENCES}

1. World Health Organisation, 2015. WHO world report on Ageing 2015. http://www.who.int/ageing/publications/world-report-2015/ en/

2. Ekerstad N, Edberg A, Carlsson P. Characteristics of multiplediseased elderly in Swedish hospital care and clinical guidelines: do they make evidence-based priority setting a "mission impossible"? International Journal of Ageing and Later Life 2009;3:71-95.

3. Ellis G, Whitehead M, O'Neill D, et al. Comprehensive geriatric assessment for older adults admitted to hospital: The Cochrane Library, 2011.

4. Stuck AE, Siu AL, Wieland GD, et al. Comprehensive geriatric assessment: a meta-analysis of controlled trials. Lancet 1993;342:1032-6.

5. Thomas EJ, Brennan TA. Incidence and types of preventable adverse events in elderly patients: population based review of medical records. BMJ 2000;320:741-4.

6. Rubenstein LZ, Josephson KR, Wieland GD, et al. Effectiveness of a geriatric evaluation unit. N Engl J Med Overseas Ed 1984;311:1664-70.

7. Ellis G, Langhorne P. Comprehensive geriatric assessment for older hospital patients. Br Med Bull 2004;71:45-59.

8. Baztán JJ, Suárez-García FM, López-Arrieta J, et al. Effectiveness of acute geriatric units on functional decline, living at home, and case fatality among older patients admitted to hospital for acute medical disorders: meta-analysis. BMJ 2009;338:b50.

9. Boult C, Boult LB, Morishita L, et al. A randomized clinical trial of outpatient geriatric evaluation and management. J Am Geriatr Soc 2001;49:351-9.

10. Ekdahl AW, Wirehn AB, Alwin J, et al. Costs and effects of an ambulatory geriatric unit (the AGe-FIT Study): a randomized controlled trial. J Am Med Dir Assoc 2015;16:497-503.

11. Ekdahl AW, Alwin J, Eckerblad J, et al. Long-term evaluation of the Ambulatory Geriatric Assessment: A Frailty Intervention Trial (AGeFIT): clinical outcomes and total costs after 36 months. J Am Med Dir Assoc 2016;17:263-8.

12. Mazya AL, Garvin P, Ekdahl AW. Outpatient comprehensive geriatric assessment: effects on frailty and mortality in old people with multimorbidity and high health care utilization. Aging Clin Exp Res. In Press. 2018.

13. Pacala J. Prevention of iatrogenic complications in the elderly, 2013.

14. Chan AW, Tetzlaff JM, Altman DG, et al. SPIRIT 2013 statement: defining standard protocol items for clinical trials. Ann Intern Med 2013;158:200-7.

15. Patientadministrative System of Skane - PASIS. https://vardgivare. skane.se/it/it-stod-och-tjanster-a-o/pasis/

16. Swedish registry of social care act desicions. https://www. socialstyrelsen.se/register/socialtjanstregister/socialtjanstinsatsertill aldreochpersonermedfunktionsnedsattning

17. Ekdahl AW, Odzakovic E, Hellström I. Living unnoticed: cognitive impairment in older people with multimorbidity. $J$ Nutr Health Aging 2016;20:1-5.

18. EuroQol Group. EuroQol - a new facility for the measurement of health-related quality of life. Health Policy 1990;16:199-208.

19. Guralnik JM, Ferrucci L, Pieper CF, et al. Lower extremity function and subsequent disability: consistency across studies, predictive models, and value of gait speed alone compared with the short physical performance battery. J Gerontol A Biol Sci Med Sci 2000;55:M221-M231.

20. Fried LP, Tangen CM, Walston J, et al. Frailty in older adults: evidence for a phenotype. J Gerontol A Biol Sci Med Sci 2001;56:M146-M157.

21. Rockwood K, Song X, MacKnight C, et al. A global clinical measure of fitness and frailty in elderly people. CMAJ 2005;173:489-95.

22. Asberg $\mathrm{KH}$, Sonn U. The cumulative structure of personal and instrumental ADL. A study of elderly people in a health service district. Scand J Rehabil Med 1989;21:171-7.

23. Nasreddine ZS, Phillips NA, Bédirian V, et al. The montreal cognitive assessment, MoCA: a brief screening tool for mild cognitive impairment. J Am Geriatr Soc 2005;53:695-9. 